\title{
Black hole goes with the flow
}

\section{A technique called reverberation mapping has previously been used to probe the structure of matter around supermassive black holes. Observations suggest that this technique can also be applied to much smaller black holes. SEE LETTER P.198}

\section{DARYL HAGGARD}

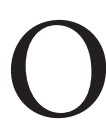
ne of the most exotic predictions of Albert Einstein's general theory of relativity was the existence of black holes, which come in two sizes. Stellar-mass black holes are typically city-sized, have masses up to ten times that of the Sun and are born from the explosions of enormous stars. Supermassive black holes are Solar System-sized, weigh millions to billions of solar masses and reside at the centres of most massive galaxies. On page 198, Kara et al. ${ }^{1}$ report observations of an astronomical object discovered last March $^{2,3}$ known as MAXI J1820 + 070, which consists of a stellar-mass black hole that is collecting (accreting) gas from a companion star through a structure called an accretion disk. These observations provide key insights into the physics of black-hole accretion.

Kara and colleagues carried out reverberation mapping, a technique that uses light to probe the geometry of matter near a black hole. To get a sense of how this technique works, imagine listening to water dripping inside a cave. First you would hear the sound of each drip, and then you would hear an echo when each sound bounces off the cave walls. The larger the cave, the longer the time delay (reverberation lag) between the drip and the echo. The sound of the drip is analogous to light that is emitted from a black hole's corona - a region of hot gas located above or below the accretion disk. The echo is akin to light from the corona that interacts with the inner edge of the accretion disk and is re-emitted.

Reverberation mapping has been used to determine the structure of matter near supermassive black holes and to measure the masses of these black holes indirectly ${ }^{4,5}$. Observations of growing supermassive black holes, known as active galactic nuclei, have led researchers to estimate reverberation lags of about $50 \mathrm{sec}-$ onds ${ }^{5}$. Such time delays indicate that some of the light emitted by the corona is re-emitted from a region within approximately 10 gravitational radii of the black hole (gravitational radii quantify the size of a black hole).

If black-hole-scaling relations hold, then, on the basis of these observations, X-ray reverberation lags for stellar-mass black holes should be of the order of milliseconds. But a few initial detections of these reverberation lags indicated
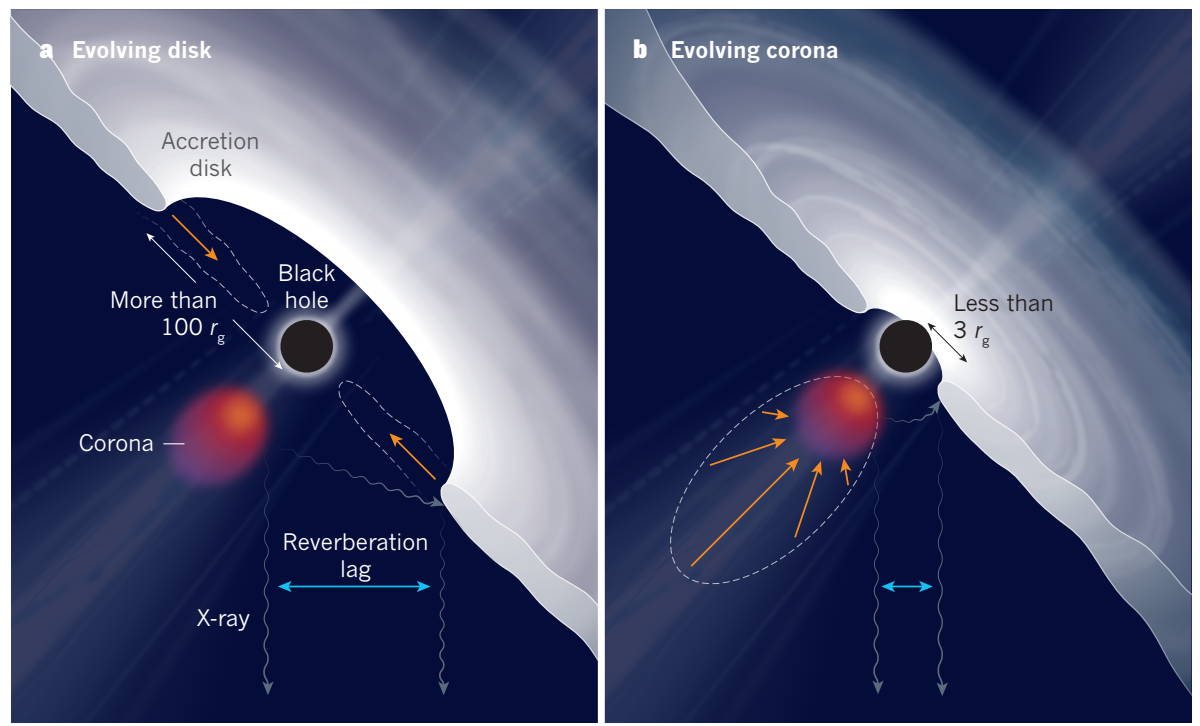

Figure 1 | Black-hole surroundings inferred from reverberation mapping. a, Stellar-mass black holes are typically accompanied by a surrounding structure called an accretion disk and a region of hot gas (a corona). A technique known as reverberation mapping can be used to probe the surroundings of a black hole by measuring the time delay (reverberation lag) between X-rays emitted from the corona and X-rays that interact with the inner edge of the accretion disk and are re-emitted. Initial observations ${ }^{6,7}$ suggested that these reverberation lags are long, implying that the accretion disk's inner edge is far from the black hole, and pointing to models in which the disk evolves over time (orange arrows). b, However, Kara et al. ${ }^{1}$ report high-resolution observations that indicate short reverberation lags. Their findings suggest that the inner disk remains close to the black hole and that the corona, rather than the disk, evolves over time. Gravitational radii $\left(r_{\mathrm{g}}\right)$ quantify the size of a black hole.

timescales about ten times longer ${ }^{6,7}$. These results led researchers to develop models that attribute the long reverberation lags to a truncated accretion disk, in which the inner edge of the disk is positioned hundreds of gravitational radii from the black hole (Fig. 1a). In these models, the accretion disk evolves over time - the truncation radius gradually becomes smaller when the black hole undergoes an outburst, owing to an increasing accretion rate. However, the initial detections ${ }^{6,7}$ were limited by the spectral and time resolution of available $\mathrm{X}$-ray instruments.

Kara et al. collected exquisite high-timeresolution X-ray spectra from the Neutron star Interior Composition Explorer (NICER $)^{8}$ installed on the International Space Station. They used these spectra to monitor changes in the reverberation signal from MAXI J1820 + 070 and to trace variations in the structure of the accretion flow as the rate of accretion underwent huge and rapid changes.
The authors detected millisecond-timescale reverberation lags during a particular outburst of X-rays from MAXI J1820 + 070. These reverberation lags mapped the distance between the corona and the inner edge of the accretion disk during the black hole's transition from outburst back to its normal state (quiescence).

The authors found that particular spectral lines that characterize the emission from the inner disk were remarkably stable during this transition. Moreover, these spectral lines indicated that the inner disk is positioned at less than three gravitational radii from the black hole (Fig. 1b). Both of these findings challenge truncated-disk models, and paint a picture of unified transitions in the accretion states of stellar-mass and supermassive black holes. The shift of the reverberation lags to shorter timescales suggests that the corona transforms from a vertically extended structure at early stages in the transition to a more compact structure at later stages. These changes occur 
on timescales that are, again, in excellent agreement with predictions from black-holescaling relations.

Many studies have explored whether there is a similarity between the accretion-state transitions in stellar-mass and supermassive black holes ${ }^{9-12}$. However, these studies have been plagued by uncertainties because of the extremely different timescales involved days to months for transitions in stellar-mass black holes, compared with tens of thousands of years for those expected in active galactic nuclei. Kara and colleagues' detection of millisecond-timescale reverberation lags during an outburst from MAXI J1820 + 070 adds a valuable piece to the puzzle, but this event represents only a single data point. The authors' findings could be strengthened in two ways.

First, other stellar-mass black holes must be studied as they transition from outburst to quiescence, using NICER and future high-time-resolution X-ray instruments that have even better sensitivity. A compiled sample of data from these outbursts, more than one of which is associated with millisecondtimescale reverberation lags, would strengthen the current findings. Second, studies of highly variable active galactic nuclei, in particular those whose supermassive black holes transition rapidly from bright to faint states, or vice versa $^{13-16}$, would provide valuable tests of whether the structures of accretion disks are similar for stellar-mass and supermassive black holes. Together, these complementary studies could provide an unprecedented look at changes that take place in the immediate surroundings of all accreting black holes.

Daryl Haggard is in the Department of Physics and at the McGill Space Institute, McGill University, Montreal, Quebec H3A 2A7, Canada. e-mail:daryl.haggard@mcgill.ca

1. Kara, E. et al. Nature 565, 198-201 (2019).

2. Shidatsu, M. et al. Astrophys. J. 868, 54 (2018).

3. Tucker, M. A. et al. Astrophys. J. Lett. 867, L9 (2018).

4. Uttley, P., Cackett, E. M., Fabian, A. C., Kara, E. \& Wilkins, D. R. Astron. Astrophys. Rev. 22, 72 (2014)

5. Kara, E. et al. Mon. Not. R. Astron. Soc. 462, 511-531 (2016).

6. Uttley, P. et al. Mon. Not. R. Astron. Soc. Lett. 414 L60-L64 (2011).

7. De Marco, B., Ponti, G., Muñoz-Darias, T. \& Nandra, K. Astrophys. J. 814, 50 (2015).

8. Gendreau, K. C. et al. Proc. SPIE 9905, 99051H (2016).

9. Merloni, A., Heinz, S. \& Di Matteo, T. Mon. Not. R. Astron. Soc. 345, 1057-1076 (2003).

10. Falcke, H., Körding, E. \& Markoff, S. Astron. Astrophys. 414, 895-903 (2004).

11.McHardy, I. M., Koerding, E., Knigge, C., Uttley, P. \& Fender, R. P. Nature 444, 730-732 (2006).

12. Körding, E. G. et al. Mon. Not. R. Astron. Soc. 380, 301-310 (2007).

13.Lamassa, S. M. et al. Astrophys. J. 800, 144 (2015).

14. Ruan, J. J. et al. Astrophys. J. 826, 188 (2016).

15.Gezari, S. et al. Astrophys. J. 835, 144 (2017).

16. Noda, H. \& Done, C. Mon. Not. R. Astron. Soc. 480 3898-3906 (2018).

\section{Designer protein delivers signal of choice}

contributes to the formation of the signalling complex, strengthening the binding between IL-2 and IL- $2 R \beta \gamma_{c}$ roughly 100 -fold. IL- 2 is used to treat some people with cancer, in whom it stimulates the cells of the immune system to work more effectively to eliminate tumour cells ${ }^{2}$. However, IL-2 has toxic side effects that limit its use $\mathrm{e}^{3,4}$. The mechanisms responsible for the toxicity are not fully understood, but studies $^{5}$ in mice indicate that the adverse effects are associated with the binding of CD25.

Several groups ${ }^{6}$ have therefore sought to engineer a variant of IL- 2 that no longer binds CD25 but still promotes the formation of IL- $2 \mathrm{R} \beta \gamma_{\mathrm{c}}$. The standard engineering approach is to start with the natural protein and find a combination of mutations that results in a variant protein with the desired binding properties. But this approach has foundered when applied to IL-2. This is partly because the unmutated protein is not very stable, and, as Silva et al. demonstrate, the mutated proteins are typically even less stable, which is problematic for the manufacture and storage of a therapeutic agent. In addition, the mutated cytokines have reduced potency
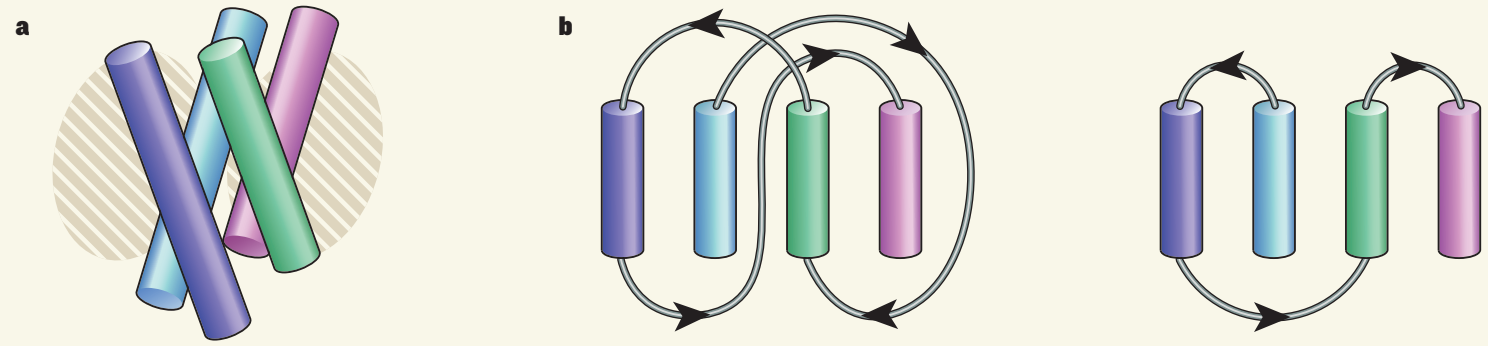

Figure 1 | Redesign for a cytokine protein. a, The cytokine protein IL-2 has potential therapeutic applications, but its clinical effectiveness is limited in part by its instability. The protein has a core of four $\alpha$-helices (shown as cylinders) connected by loops (not shown), and exerts its biological effects by forming interactions with target receptors at two regions (shaded areas) on its surface. $\mathbf{b}$, Silva et al. ${ }^{1}$ used a computational strategy to redesign IL-2.
They fixed the relative positions of the receptor-binding surfaces and then designed a stable four-helix bundle to present these surfaces to receptors. The designer protein is radically different in structure from the natural cytokine. For example, the connectivity of the four helices in the natural cytokine (left) is completely different from that of the analogous helices in the designer protein (right). 